\title{
遥感反演离散植被有效叶面积指数的 空间尺度效应
}

\author{
范闻捷"，盖颖颖，徐希孺，问涁彦 \\ 北京大学遥感与地理信息系统研究所, 北京 100871 \\ *E-mail: fanwj@pku.edu.cn
}

收稿日期: 2012-07-02; 接受日期: 2012-09-14

国家自然科学基金(批准号: 91025006, 40871186, 40730525)、国家重点基础研究发展计划(编号: 2007CB714402)和国家高技术研究发展计 划(编号: 2009AA12Z143, 2009AA122103)资助

\begin{abstract}
摘要叶面积指数(LAI)是表征植被几何结构和生长状态的关键生物物理参数, 也是 气候、能量和碳循环等模型的重要输入参数. 遥感反演 LAI 的尺度效应问题一直备受 关注. 考虑到尼尔逊参数对离散植被的 BRDF 模型的影响, 我们定义有效叶面积指数 $\mathrm{LAI}_{\mathrm{eff}}, \mathrm{LAI}_{\mathrm{eff}}$ 与传统概念下的叶面积指数具有相同的表征植被冠层内叶子浓密程度的 主要功能, 因此, 本文以讨论有效叶面积指数为主. 离散植被的空间尺度效应产生机 理与连续植被有所不同，本文在方向性二阶微分方法(DSD)反演有效叶面积指数的基 础上, 讨论了离散植被空间尺度效应产生的原因, 并提出了有效叶面积指数的尺度转 换公式. 理论分析结果表明高分辨率像元有效叶面积指数反演值的平均值总是大于等 于同一目标低分辨率像元的有效叶面积指数反演值. 另外, 本文基于实际航空高光谱 遥感影像, 反演了中国河北省张家口市怀来县某植被覆盖区的有效叶面积指数, 其反 演结果验证了该结论的正确性和尺度转换公式的可靠性.
\end{abstract}

关键词

空间尺度效应

离散植被

有效叶面积指数

方向性二阶微分方法
叶面积指数是表征植被结构和生长状态的关键 参数, 影响着植被许多生物、物理过程, 如光合、呼 吸、蒸腾、碳循环和降水截获量估算等, 也是很多陆 面模型的重要输入变量 ${ }^{[1 \sim 4]}$. 精确反演 LAI 是评估植 被长势和固碳能力的重要前提, 对维持生态平衡和 提高人类的生产生活水平具有深远意义. 遥感影像 可以提供不同空间尺度的遥感信息, 经过反演可以 得到不同分辨率的各类 LAI 产品. Chen 和 Black ${ }^{[5]}$ 提 出, 根据光学原理反演得到的 $\mathrm{LAI}$ 为有效 $\mathrm{LAI}\left(\mathrm{LAI}_{\mathrm{eff}}\right)$,
因为大多数模型都忽略了冠层内的叶片非均匀分布 特征. 因此目前大多数 LAI 遥感产品, 如 SPOT-4 $\operatorname{VEGETATION}(\mathrm{VGT})$ 产品假设尼尔逊参数为 $1^{[6]}$, 没 有考虑冠层的群聚效应, 其 LAI 应为 $\mathrm{LAI}_{\text {eff }}$ - $\mathrm{LAI}_{\mathrm{eff}}$ 同样具有相同的表征植被冠层内叶子浓密程度的主 要功能.

研究证明, 由不同分辨率遥感数据获得的各类 LAI 是不同的，导致用 LAI 地面测量值验证不同分辨 率遥感产品的困难 ${ }^{[7 ~ 13]}$. 国内外针对遥感反演 LAI 的

中文引用格式: 范闻捷, 盖颖颖, 徐希孺, 等. 遥感反演离散植被有效叶面积指数的空间尺度效应. 中国科学: 地球科学, 2013, 43: 280-286

英文引用格式: Fan W J, Gai Y Y, Xu X R, et al. The spatial scaling effect of the discrete-canopy effective leaf area index retrieved by remote sensing. Science China: Earth Sciences, 2012, doi: 10.1007/s11430-012-4554-5 
尺度效应已进行了广泛的研究, 如 Milne 与 Cohen ${ }^{[14]}$ 对 MODIS 反演的连续植被变量, 包括 LAI 等, 进行 了多尺度分析; 陈健等 ${ }^{[15]}$ 利用 $\mathrm{TM}$ 和 MODIS 遥感数 据对大面积芦苇的 LAI 反演误差进行了分析, 并探 讨了 LAI 尺度效应产生的主要原因; Zhu 等 ${ }^{[16]}$ 采用双 层冠层反射率模型迭代运算反演成熟期小麦和玉米 的 LAI, 对 LAI 反演的尺度效应进行了定量分析, 并 从模型的非线性和地表景观结构的空间异质性两个 方面分析了引起反演误差的原因; 徐希孺等 ${ }^{\left[{ }^{[17}\right.}$ 建立 了连续植被不同尺度之间 LAI 反演值的转换公式, 证明了混合像元中连续植被所占面积比例随尺度呈 非线性变化是造成尺度效应的主要根源. 这些研究 大多针对空间分布较为均匀、个体特征不明显的连续 植被.

虽然空间异质性与反演方程的非线性是不同植 被类型产生反演各类 LAI 尺度效应的共同根源, 但 是离散植被的结构特征与连续植被有所不同, 混合 像元中连续植被所占的平均面积比例 $a_{v}(n)$ 与像元尺 度级数之间呈非线性关系, 是构成连续植被反演 LAI 存在尺度效应的主要原因. 连续植被生长空间变化 有限, 所造成的尺度效应也是有限的. 而离散植被与 此相反, 几乎每一个像元都可以视为混合像元, LAI 的空间变化成为产生遥感反演各类 LAI 尺度效应的 主导因素, 显然空间结构上的差异必然导致尺度转 换规律的不同, 讨论离散植被遥感反演 LAI 的尺度 效应是十分必要的. 本文以离散植被 $\mathrm{LAI}_{\mathrm{eff}}$ 反演值的 尺度效应和尺度纠正为主要研究内容, 在方向性二 阶微分方法反演有效叶面积指数的基础上, 力图从 理论上分析离散植被尺度效应产生的机理, 并得到 尺度转换公式.

\section{1 离散植被 $\mathrm{LAI}_{\text {eff }}$ 尺度效应的遥感反演公式}

LAI 普遍定义为地表单位面积柱体内叶子表面 积的一半与地表面积之比 ${ }^{[5]}$, 该定义中没有对植被柱 体的边界和叶面积的空间分布做严格的界定. 混合 像元一般是由植被和异质组成的, 假设离散植被混 合像元如图 1 所示, 像元内分布着球形树冠, 树冠大 小不一, 若 $A$ 为混合像元大小, $a_{1}$ 为所有树冠的垂直 投影面积之和, $a_{2}$ 为异质部分, $A=a_{1}+a_{2}$.

则像元平均叶面积指数 $\mathrm{LAI}_{\mathrm{a}}$ 的定义为

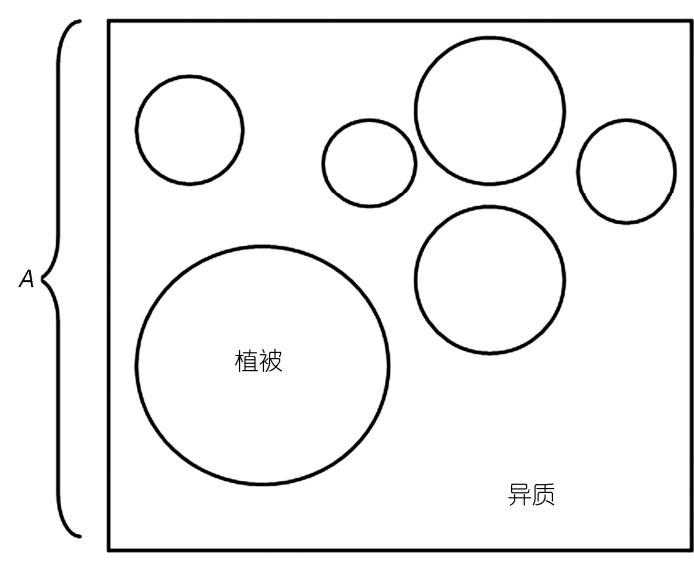

图 1 离散植被混合像元示意图

$$
\mathrm{LAI}_{\mathrm{a}}=\frac{L A}{A},
$$

其中, $L A$ 为像元柱体内植被叶子总面积的一半.

植被对外来辐射之反射在 $2 \pi$ 空间中呈二向性反 射特征, 其反射率可近似表达为一次散射项 $\rho^{1}\left(\Omega_{i}, \Omega_{v}\right)$ 与多次散射项 $\rho^{m}\left(\Omega_{i}, \Omega_{v}\right)$ 之和, 即

$$
\rho\left(\Omega_{i}, \Omega_{v}\right) \cong \rho^{1}\left(\Omega_{i}, \Omega_{v}\right)+\rho^{m}\left(\Omega_{i}, \Omega_{v}\right),
$$

其中, $\Omega_{i}$ 和 $\Omega_{v}$ 分别代表太阳直射方向与观测方向, $\rho^{1}$ 为二向性反射主要来源, $\rho^{m}$ 呈近似各向同性.

按照几何光学模型对离散植被一次反射项的表 述 ${ }^{[18 \sim 21]}$, 传感器视场中存在光照与阴影条件下的树 冠、光照与阴影条件下的背景, 它们在视场中所占面 积比例可分别表达为 $K_{c}, K_{t}$ 与 $K_{g}, K_{z}$, 且满足 $K_{c}+K_{t}+K_{g}+K_{z}=1$, 传感器所接收到的辐射亮度 $L$ 可表 达为

$$
L=K_{c} L_{c}+K_{t} L_{t}+K_{g} L_{g}+K_{z} L_{z},
$$

其中假定四分量为均匀的朗伯面, $L_{c}, L_{t}$ 与 $L_{g}, L_{z}$ 分别 为它的漫反射亮度值. 当传感器在 $2 \pi$ 空间中变换位 置时, $K_{c}, K_{t}, K_{g}, K_{z}$ 随之改变, 这就是离散植被二向性 反射产生的主要根源. 本文对此做了一个改动, 把立 足点由树冠移至树冠元素(主要是树叶), 四分量便成 为光照和阴影条件下的叶子、光照和阴影条件下的背 景, 所用符号保持不变, 此时视线方向上的透过率 $P\left(\Omega_{v}\right)$ 就十分重要, 因为它成为 $K_{c}+K_{t}$ 与 $K_{g}+K_{z}$ 之间 的分界线. 对均匀连续植被而言，方位方向上拥有近 似的一致性, 故

$$
P\left(\theta_{v}\right)=\exp \left(-\frac{G_{v}}{\mu_{v}} \mathrm{LAI}_{\mathrm{a}}\right),
$$


其中 $G_{v}$ 为 $v$ 方向上的 $G$ 函数值, $\mu_{v}=\cos \theta_{v}$. 如果把 离散植被视为连续植被在树冠尺度上发生了群聚的 结果, 根据 $\mathrm{Nilson}^{[22]}$ 对群聚植被平均透过率的表述 方式, 则离散植被像元的平均透过率为

$$
P\left(\theta_{v}\right)=\exp \left(-\frac{G_{v}}{\mu_{v}} \lambda_{v} \mathrm{LAI}_{\mathrm{a}}\right) .
$$

此处 $\lambda$ 为尼尔逊参数, 也被称为聚集指数 (Clumping index), $0<\lambda<1$, 这种表达方式的关键在于如何获知 $\lambda$. 如果离散植被冠层的平均透过率、平均叶面积指数、 $G_{v}$ 值可以通过实测而知, 则 $\lambda$ 可由公式(5)算得, 但不 可能对每一个像元都获得如此众多的实测值, 这给 遥感反演平均叶面积指数带来困难.

至于 $K_{c}$ 与 $K_{t}$ 以及 $K_{g}$ 与 $K_{z}$ 之间分割的表达可采 用推广的 Kuusk 模型 ${ }^{[23,24]}$, 则离散植被的一次散射 $\rho^{1}\left(\Omega_{i}, \Omega_{v}\right)$ 可表达为

$$
\begin{aligned}
\rho^{1}\left(\Omega_{i}, \Omega_{v}\right)= & (1-\beta) K_{c} \rho_{\text {leaf }}+\beta K_{t} \rho_{\text {leaf }} \\
& +(1-\beta) K_{g} \rho_{\text {soil }}+\beta K_{z} \rho_{\text {soil }},
\end{aligned}
$$

其中,

$$
\begin{aligned}
K_{c}= & 1-\exp \left(-\sqrt{\frac{G_{i} G_{v} \lambda_{i} \lambda_{v}}{\mu_{i} \mu_{v}}} w \mathrm{LAI}_{\mathrm{a}}\right), \\
K_{t}= & \exp \left(-\sqrt{\frac{G_{i} G_{v} \lambda_{i} \lambda_{v}}{\mu_{i} \mu_{v}}} w \mathrm{LAI}_{\mathrm{a}}\right)-\exp \left(-\frac{G_{v} \lambda_{v}}{\mu_{v}} \mathrm{LAI}_{\mathrm{a}}\right), \\
K_{g}= & \exp \left[-\left(\frac{\lambda_{i} G_{i}}{\mu_{i}}+\frac{\lambda_{v} G_{v}}{\mu_{v}}-\sqrt{\frac{G_{i} G_{v} \lambda_{i} \lambda_{v}}{\mu_{i} \mu_{v}}} w\right) \mathrm{LAI}_{\mathrm{a}}\right], \\
K_{z}= & \exp \left(-\frac{G_{v} \lambda_{v}}{\mu_{v}} \mathrm{LAI}_{\mathrm{a}}\right) \\
& -\exp \left[-\left(\frac{\lambda_{i} G_{i}}{\mu_{i}}+\frac{\lambda_{v} G_{v}}{\mu_{v}}-\sqrt{\frac{G_{i} G_{v} \lambda_{i} \lambda_{v}}{\mu_{i} \mu_{v}}} w\right) \mathrm{LAI}_{\mathrm{a}}\right], \\
\beta= & \frac{E}{\mu_{i} F+E}, \\
w= & \frac{1}{H} \int_{0}^{H} \mathrm{e}^{-\frac{z \delta}{d}} \mathrm{~d} z=\frac{d}{H \delta}\left(1-\mathrm{e}^{-\frac{H \delta}{d}}\right),
\end{aligned}
$$

$E$ 为天空漫辐射对地表构成的辐照度, $\mu_{i}=\cos \theta_{i}, \theta_{i}$ 为太阳天顶角, $\mu_{i} F$ 为太阳直射对地表构成的辐照度, $\lambda_{i}$ 与 $\lambda_{v}$ 分别代表太阳入射和视线方向上的尼尔逊参 数. $H$ 为冠层厚度, $\delta=\sqrt{\frac{1}{\mu_{i}^{2}}+\frac{1}{\mu_{v}^{2}}-\frac{2 \cos \alpha}{\mu_{i} \mu_{v}}}, \alpha$ 为视
线与射线间的夹角, $d$ 为与叶子形状和尺度有关的经 验参数. $\rho_{\text {leaf }}$ 与 $\rho_{\text {soil }}$ 分别代表叶子的漫反射率与土壤 或异质背景的漫反射率，此处忽略了叶片及异质背 景表面的非各向同性反射特性，假定为朗伯表面.

公式(6)表明 $\rho^{1}\left(\Omega_{i}, \Omega_{v}\right)$ 是一个多元函数, 除了 $\mathrm{LAI}_{\mathrm{a}}$ 之外, 还有异质背景反射率大小, 传感器-目标太阳三者几何关系以及 $\beta$ 值等都成为影响 $\rho^{1}$ 值的因素. 显然, 要讨论遥感反演 $\mathrm{LAI}_{\mathrm{a}}$ 的尺度效应, 就必须排 除 $\mathrm{LAI}_{\mathrm{a}}$ 之外所有其他因素的干扰, 所得的函数关系 才是真正的尺度转换公式. 否则除尺度之外的其他 因素均可导致 $\mathrm{LAI}_{\mathrm{a}}$ 反演值的变化, 这与尺度效应的 概念相背离. 为此反演离散植被 $\mathrm{LAI}_{a}$ 的 DSD 方法是 一种较为合适的方法.

(1) 通过 $\rho^{1}\left(\Omega_{i}, \Omega_{v}\right)$ 对波段的二阶微商, 在 $0.68 \sim 0.71 \mu \mathrm{m}$ 范围内 $\rho_{\text {leaf }}^{\prime \prime}>>\rho_{\text {soil }}^{\prime \prime}$, 且这一结果可以推 广到水体、城镇和沥青马路等其他物体，这一特性可 以广泛地用于滤除背景干扰 ${ }^{[25]}$

（2）根据晴空条件下的实测数据(能见度大于 25 $\mathrm{km}), 0.7 \mu \mathrm{m}$ 附近 $\beta$ 的平均值约为 0.01 , 所以对 0.68 0.71 $\mu \mathrm{m}$ 范围内的二阶微商而言, 作为一级近似, 所有阴影面的贡献可以忽略不计 ${ }^{[26]}$.

(3) 当波长 $\lambda<0.73 \mu \mathrm{m}$ 时, 多次散射项 $\rho^{m}\left(\Omega_{i}, \Omega_{v}\right)$ 可以忽略 ${ }^{[25]}$. 在 $0.68 \sim 0.71 \mu \mathrm{m}$ 范围内, $\rho^{m}\left(\Omega_{i}, \Omega_{v}\right)$ 的波段二阶微商与 $\rho^{1}\left(\Omega_{i}, \Omega_{v}\right)$ 的二阶微商 之比小于 0.05 .

(4) 二阶微商能部分消除大气效应 ${ }^{[27,28]}$. 令

$$
x=\frac{\rho^{1^{\prime \prime}}}{\rho_{\text {leaf }}^{\prime \prime}}=1-\exp \left(-\sqrt{\frac{G_{i} G_{v} \lambda_{i} \lambda_{v}}{\mu_{i} \mu_{v}}} w \mathrm{LAI}_{\mathrm{a}}\right),
$$

且令有效叶面积指数 $\mathrm{LAI}_{\text {eff }}=\sqrt{\lambda_{i} \lambda_{v}} \mathrm{LAI}_{\mathrm{a}}$, 则

$$
x \cong 1-\exp \left(-\sqrt{\frac{G_{i} G_{v}}{\mu_{i} \mu_{v}}} w \mathrm{LAI}_{\text {eff }}\right) .
$$

(5) $\rho^{1 "}$ 由遥感实测得到, $\rho_{\text {leaf }}^{\prime \prime}$ 由数据库获得, $G_{i}$ 和 $G_{v}$ 可由数据库或实测获得, 唯有每个像元的 $\lambda_{i}$ 与 $\lambda_{v}$ 值目前还没有有效的获知方法，所以引入有效 叶面积指数，可以暂时回避这一困难，同时有效叶面 积指数代表着主要起光合作用的那部分叶面积, 可 直接用于光合效率计算等.

遥感可测值 $x$ 与有效叶面积指数之间的函数关 系可用图 2 表达 ${ }^{[25]}$, 每一条曲线代表 $\alpha$ 取不同值, $\alpha$ 取 


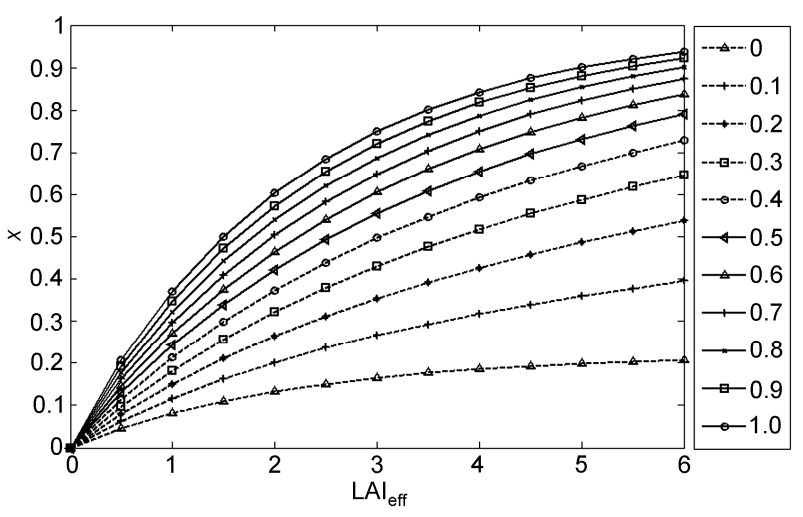

图 2 不同观测几何条件下的遥感可测值 $x$ 与有效叶面积 指数的函数关系

不同曲线代表不同的 $\alpha$, 即不同的太阳-目标-传感器几何关系

“0”代表在热点处观测, 由此可见, 太阳-目标-传感器 三者几何关系的不同并没有使 $\mathrm{LAI}_{\mathrm{eff}}$ 的反演规律发生 本质上的变化, 只是使反演 $\mathrm{LAI}_{\mathrm{eff}}$ 的能力随 $\alpha$ 的增大 而有所下降(热点处 $\mathrm{LAI}_{\text {eff }}$ 的动态范围最大, 随 $\alpha$ 增大, $\mathrm{LAI}_{\mathrm{eff}}$ 的饱和点不断下降), 所以如果在热点处讨论 LAI 的尺度效应, 便可以很方便地推广至其他方向. 在对高光谱图像进行大气纠正和滤波处理后, 对反 演 $\mathrm{LAI}_{\text {eff }}$ 起干扰作用的因子都得到了妥善处理.

公式(7)便是基于 DSD 方法的反演离散植被有效 叶面积指数 $\mathrm{LAI}_{\mathrm{eff}}$ 的出发方程. 目前国际上正热烈讨 论针对离散植被实测 $\lambda$ 值的各种方法, 有研究利用热 点与冷点反射率遥感反演 $5 \mathrm{~km}$ 像元的尼尔逊参数 ${ }^{[29]}$, 但大多数遥感产品仍未考虑群聚效应.

\section{2 遥感反演离散植被有效叶面积指数的尺 度效应}

\section{1 遥感反演离散植被有效叶面积指数产生尺度 效应的原因}

尺度效应可以表述为对下述问题的回答，“大像 元的有效叶面积指数反演值是否等于同一对象的小 像元反演值的线性平均值”, 如果答案为 “是”, 则称 为无尺度效应，反之, 则表明存在尺度效应，根据公 式(7)有

$$
\mathrm{LAI}_{\text {eff }}=-\sqrt{\frac{\mu_{i} \mu_{v}}{G_{i} G_{v}}} \frac{1}{w} \ln (1-x) .
$$

需要回答的问题可表述为

$$
\mathrm{LAI}_{\text {eff,A }}=? \frac{1}{n} \sum_{i=1}^{n} \mathrm{LAI}_{\text {eff, } \mathrm{a}}^{\mathrm{i}},
$$

其中, $\mathrm{LAI}_{\mathrm{eff}, \mathrm{A}}$ 代表大像元 $A$ 的有效叶面积指数反演 值, $\mathrm{LAI}_{\mathrm{eff}, \mathrm{a}}^{\mathrm{i}}$ 代表第 $i$ 个小像元 $a$ 的有效叶面积指数反 演值. 公式(8)与公式(9)相结合, 得到

$$
\sqrt{\frac{\mu_{i} \mu_{v}}{G_{i} G_{v}}} \frac{1}{w} \ln \left(1-x_{A}\right)=? \frac{1}{n} \sum_{i=1}^{n}\left[\sqrt{\frac{\mu_{i} \mu_{v}}{G_{i} G_{v}}} \frac{1}{w^{i}} \ln \left(1-x_{a}^{i}\right)\right],
$$

其中, $x_{A}=\frac{1}{n} \sum_{i=1}^{n} x_{a}^{i}$. 若假设像元内树冠空间分布均 匀, 则有 $\sum_{i=1}^{n} x_{a}^{i}=n x_{a}^{i}, w=w^{i}$, 等式(10)的左边与右边 相等. 换言之, 离散植被树冠在空间中均匀分布, 其 有效叶面积指数遥感反演值将不出现尺度效应, 即 离散植被空间分布不均一是产生遥感反演离散植被 有效叶面积指数尺度效应的必要条件.

\section{2 离散植被有效叶面积指数反演值的尺度转换} 公式

设大像元 $A$ 由 $n$ 个小像元组成, 每个小像元的 有效叶面积指数反演值可表达为平均值 $\mathrm{LAI}_{\mathrm{eff}, \mathrm{a,a}}$ 和涨 落值 $\Delta \mathrm{LAI}_{\mathrm{eff}, \mathrm{a}}^{\mathrm{i}}$ 之和, 即

$$
\mathrm{LAI}_{\text {eff,a }}^{\mathrm{i}}=\mathrm{LAI}_{\text {eff,a,a }}+\Delta \mathrm{LAI}_{\text {eff,a }}^{\mathrm{i}} \text {, }
$$

则有

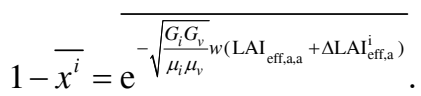

其中, 符号上面的横线代表在大像元 $A$ 范围内求平均.

鉴于 $\mathrm{LAI}_{\text {eff,a,a }}$ 与 $\Delta \mathrm{LAI}_{\text {eff,a }}^{\mathrm{i}}$ 统计不相关, 下式成立

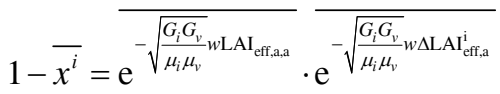

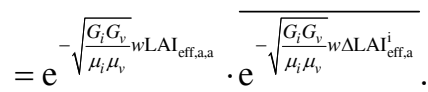

对公式(13)等号右边第二项进行泰勒级数展开, 且 $\Delta \mathrm{LAI}_{\mathrm{eff}, \mathrm{a}}^{\mathrm{i}}$ 的奇次方平均值为零, 故下式成立

$$
\begin{aligned}
\mathrm{LAI}_{\mathrm{eff}, \mathrm{A}}= & \mathrm{LAI}_{\mathrm{eff}, \mathrm{a}, \mathrm{a}}-\sqrt{\frac{\mu_{i} \mu_{v}}{G_{i} G_{v}}} \cdot \frac{1}{w} \\
& \cdot \ln \left[1+\frac{1}{2 !}\left(\frac{G_{i} G_{v}}{\mu_{i} \mu_{v}}\right) w^{2} \overline{\left(\Delta \mathrm{LAI}_{\mathrm{eff}, \mathrm{a}}\right)^{2}}\right. \\
& \left.+\frac{1}{4 !}\left(\frac{G_{i} G_{v}}{\mu_{i} \mu_{v}}\right)^{2} w^{4} \overline{\left(\Delta \mathrm{LAI}_{\mathrm{eff}, \mathrm{a}}\right)^{4}}+\cdots\right] .
\end{aligned}
$$


公式(14)等号右边第二项为“正”值，这表明对离 散植被而言, 小尺度像元遥感反演有效叶面积指数 值的平均值永远大于同一目标大尺度像元遥感反演 有效叶面积指数值, 其差值由公式(14)等号右边第二 项表达.

对于上述结果可做如下的物理解释:

在有效叶面积指数反演方法中, 信息量主要来 自于叶子的光照面对外来辐射的反射, 植被冠层的 透过率为主要影响因素, 而透过率可表达为 $e^{-x}$ 函数 形式, 如图 3 所示.

设 $\bar{x}$ 为平均值, 它的左右两边等概率地出现 $+\Delta x$ 与 $-\Delta x$, 它们所对应的透过率值分别为 $p_{1}$ 与 $p_{2}$, 其平均值为 $p_{3}, p_{3}$ 所对应的 $x$ 即为反演值, 可用 $x^{r}$ 表 示, 图 3 清楚地表明反演公式的非线性是如何使 $x^{r}<\bar{x}$ 的.

总之, 遥感反演离散植被有效叶面积指数出现 尺度效应的内在原因是植被空间分布的不均一性 (出现 $\pm \Delta x)$ 及反演公式的非线性 $\left(e^{-x}\right)$, 两个条件缺 一不可.

\section{3 实验验证}

\section{1 研究区与实验数据}

本次研究所选择区域为中国河北省张家口市怀 来县境内官厅水库附近的植被覆盖区 $\left(115^{\circ} 46^{\prime}\right.$ $\left.115^{\circ} 52^{\prime} \mathrm{E}, 40^{\circ} 23^{\prime} \sim 40^{\circ} 27^{\prime} \mathrm{N}\right)$, 该区属华北沿海平原向 内蒙古高原过渡地带, 为温带大陆性季风气候区, 冬

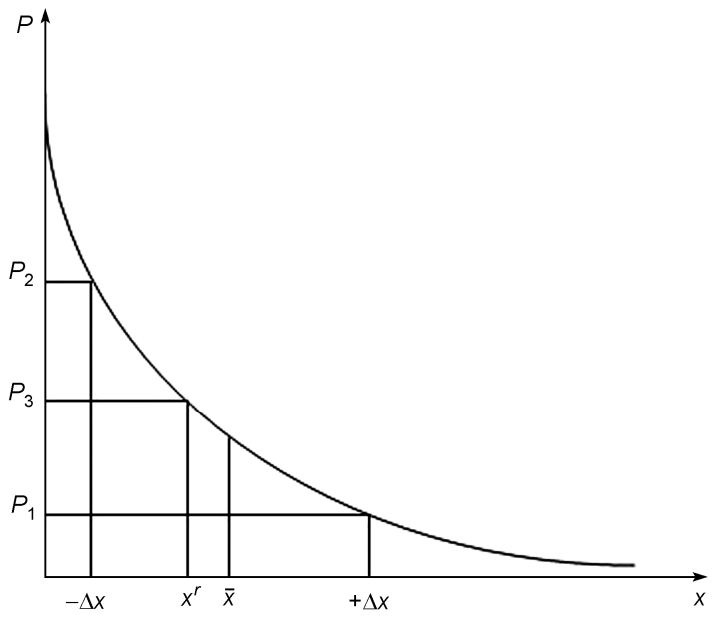

图 3 离散植被有效叶面积指数反演值尺度效应示意图
季寒冷干燥, 夏季炎热多雨 ${ }^{[0,31]}$. 研究区内植被类型 丰富多样，地面草种多为苇状羊茅和碱茅，并随机分 布有大量落叶乔木，如旱柳、垂柳和杨树等.

实验数据采用 2010 年 9 月 23 日覆盖该研究区的 航空遥感影像, 获取图像的高光谱传感器为芬兰 SPECIM 公司在 AisaEAGLET 基础上根据项目要求 的技术指标而制成，光谱范围为 400 1000 $\mathrm{nm}$, 光谱 分辨率为 $3.3 \mathrm{~nm}$, 空间分辨率为 $0.8 \mathrm{~m}$. 所选择实验 验证区域的位置及其真彩色合成影像如图 4 所示.

首先对获取的图像进行几何、辐射和大气校正, 辐射和几何校正采用 CaliGeo 软件, 大气校正采用 ENVI 的 FLAASH 模块. 由于遥测数据中不可避免地 会混合多种高频噪声，而微分运算对高频变化极为 敏感，所以方向性二阶微分方法受噪声干扰较大，因 此反演有效 LAI 之前要先进行滤波, 采用 MNF 变换 消除高光谱数据中的噪声, 基本过程是先对图像进 行正向 MNF 变换, 去除噪声较大的波段, 保留含有 较多有用信息的波段, 再进行反向 MNF 变换 ${ }^{[32]}$.

为了验证离散植被尺度效应及获得不同分辨率 之间有效 LAI 的尺度转换关系，需保证不同分辨率 图像之间除像元尺度不同外，其它影响 LAI 反演的 因素都要去除. 因此，在 AisaEAGLET 高分辨率影像

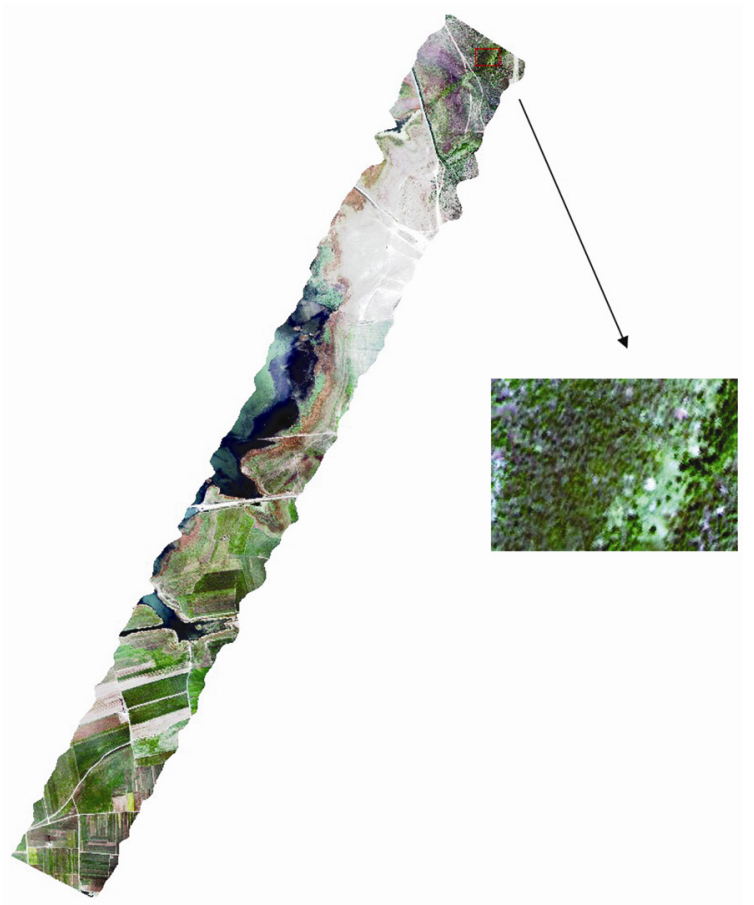

图 4 实验验证区域地理位置及其真彩色合成影像 
的基础上, 对像元的辐射亮度求线性平均得到低分 辨率影像，低分辨率影像的空间分辨率为 $8 \mathrm{~m}$.

\section{2 实验验证结果}

利用方向性二阶微分方法分别反演高分辨率和 低分辨率影像中像元的有效 LAI 值, 验证低分辨率 像元的有效 LAI 反演值与高分辨率像元有效 LAI 反 演值的平均值的关系如图 5 所示. 可见, 对于离散植 被而言, 大像元有效 LAI 反演值总是小于小像元有 效 LAI 反演值的平均值, 这与公式(14)所表达的含义 亦相符.

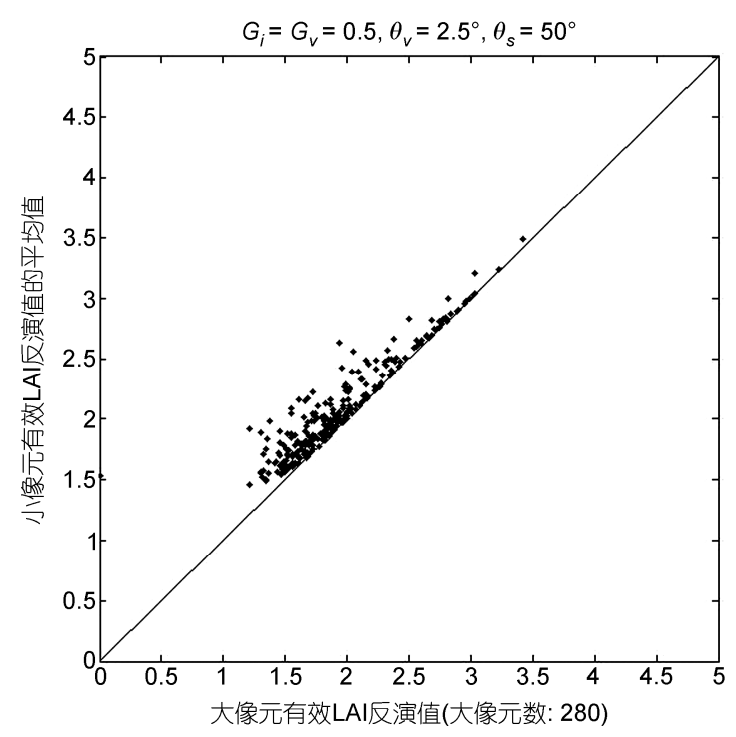

图 5 低分辨率像元的有效 LAI 反演值与高分辨率像元有 效 LAI 反演值的平均值的验证结果

差值的计算, 关键在于计算 $\overline{\left(\Delta \mathrm{LAI}_{\mathrm{eff}, \mathrm{a}}\right)^{2}}$, 小像元 有效叶面积指数 $\mathrm{LAI}_{\text {eff,a }}^{\mathrm{i}}$ 可以反演得到, 所以每个小像 元的 $\Delta \mathrm{LAI}_{\mathrm{eff}, \mathrm{a}}^{\mathrm{i}}$ 可以通过公式(11)计算得到, 代入公式 (14) 等号右边第二项, 比较该项计算结果与 $\mathrm{LAI}_{\text {eff,a, }}-\mathrm{LAI}_{\text {eff,A }}$ 的差异, 如图 6 所示, 统计显示, 通 过公式(14)等号右边第二项计算得到的小像元有效叶 面积指数反演值的平均与大像元有效叶面积指数反演 值的差值与实际差值相比, 其相对误差约为 $4.66 \%$.

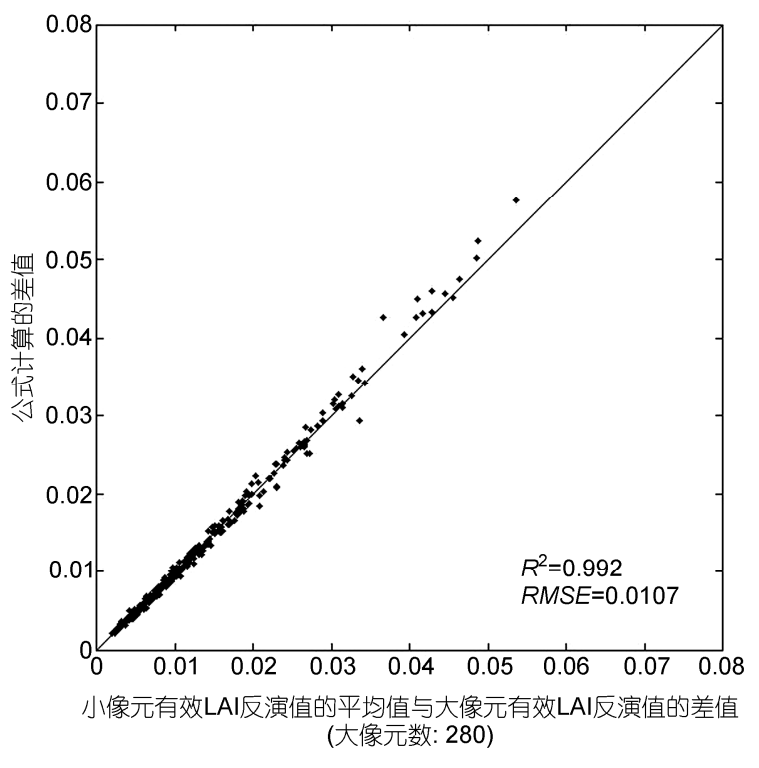

图 $6 \mathrm{LAI}_{\mathrm{eff}, \mathrm{a,a}}-\mathrm{LAI}_{\mathrm{eff}, \mathrm{A}}$ 与公式(14)等号右边第二项的验证 结果

\section{4 讨论与结论}

本文对离散植被反演有效叶面积指数尺度效应 的讨论, 再一次证实了叶面积指数空间分布不均一 与反演函数的非线性本质是决定遥感反演叶面积指 数出现尺度效应的充分必要条件, 与遥感界普遍接 受的观点一致. 但在充分肯定遥感反演叶面积指数 尺度效应共因的同时，也应充分认识到植被类型对 尺度效应机制的影响. 对连续植被而言, $\Delta \mathrm{LAI}$ 主要 出现在混合像元中, 而混合像元出现在连续植被与 异质块的交界线上, 所以当像元尺度逐渐减小时, 混 合像元数目在总像元数目中的比例会随之减少, 直至 某个像元尺度 $r_{0}$, 混合像元所占比例可以忽略不计, 这便导致植被覆盖度 $\left(a_{v}\right)$ 的平均值随像元尺度减小以 负指数形式增加. 而离散植被则完全不同, 每一个像 元都可以当作混合像元来看待, 像元间的 LAI 值起 伏明显, 因此只要引入平均叶面积指数概念, 像元的 $\Delta \mathrm{LAI}$ 值便可直接引入公式(14)计算尺度效应纠正值. 由此可见, 虽然不同植被类型其遥感反演叶面积指 数出现尺度效应的机理是相同的, 但尺度转换公式 会因造成 $\Delta \mathrm{LAI}$ 的具体过程不同而有所差异. 


\section{参考文献}

1 Bicheron P, Leroy M. A method of biophysical parameter retrieval at global scale by inversion of a vegetation reflectance model. Remote Sens Environ, 1999, 67: 251-266

2 Walthall C L, Dulaney W P, Anderson M, et al. Alternative approaches for estimating leaf area index (LAI) from remotely sensed satellite and aircraft imagery. In: Gao W, Shaw D R, eds. Proceedings of the Society of Photo-optical Instrumentation Engineers, 2004. Bellingham: SPIE-INT Soc Optical Engineering, 2004. 241-255

3 Wang Y J, Woodcock C E, Buermanna W, et al. Evaluation of the MODIS LAI algorithm at a coniferous forest site in Finland. Remote Sens Environ, 2004, 91: 114-127

4 张仁华. 定量热红外遥感模型及地面实验基础. 北京: 科学出版社, 2009. 172-176

5 Chen J M, Black T A. Defining leaf area index for non-flat leaves. Plant Cell Environ, 1992, 15: 421-429

6 Pisek J, Chen J M. Comparison and validation of MODIS and VEGETATION global LAI products over four BigFoot sites in North America. Remote Sens Environ, 2007, 109: 81-94

7 刘艳, 王锦地, 周红敏, 等. 黑河中游试验区不同分辨率 LAI 数据处理、分析和尺度转换. 遥感技术与应用, 2010, 25: 805-812

8 Chen J M. Spatial scaling of a remotely sensed surface parameter by contexture. Remote Sens Environ, 1999, 69: 30-42

9 Garrigues S, Allard D, Baret F, et al. Influence of landscape spatial heterogeneity on the non-linear estimation of leaf area index from moderate spatial resolution remote sensing data. Remote Sens Environ, 2006, 105: 286-298

10 Tian Y H, Woodcock C E, Wang Y J, et al. Multiscale analysis and validation of the MODIS LAI product I. Uncertainty assessment. Remote Sens Environ, 2002, 83: 414-430

11 Jin Z, Tian Q, Chen J M, et al. Spatial scaling between leaf area index maps of different resolutions. J Environ Manage, 2007, 85: 628-637

12 张万昌, 钟山, 胡少英. 黑河流域叶面积指数(LAI)空间尺度转换. 生态学报, 2008, 28: 2495-2503

13 张仁华, 田静, 李召良, 等. 定量遥感产品真实性检验的基础与方法. 中国科学: 地球科学, 2010, 40: 211-222

14 Milne B T, Cohen W B. Multiscale assessment of binary and continuous landcover variables for MODIS validation, mapping, and modeling applications. Remote Sens Environ, 1999, 70: 82-98

15 陈健, 倪绍祥, 李静静, 等. 植被叶面积指数遥感反演的尺度效应及空间变异性. 生态学报, 2006, 26: 1502-1508

16 Zhu X H, Feng X M, Zhao Y S, et al. Scale effect and error analysis of crop LAI inversion. J Remote Sens, 2010, 14: 579-592

17 徐希孺, 范闻捷, 陶欣. 遥感反演连续植被叶面积指数的空间尺度效应. 中国科学 D 辑: 地球科学, 2009, 39: 79-87

18 Li X W, Strahler A H. Geometric-optical modeling of a conifer forest canopy. IEEE Trans Geosci Remote Sensing, 1985, 23: 705-721

19 Li X W, Strahler A H. Geometric-optical bidirectional reflectance modeling of a conifer forest canopy. IEEE Trans Geosci Remote Sensing, 1986, GE-24: 906-919

20 Chen J M, Leblanc S G. A four-scale bi-directional reflectance model based on canopy architecture. IEEE Trans Geosci Remote Sensing, 1997, 35: 1316-1337

21 徐希孺. 遥感物理. 北京: 北京大学出版社, 2005. 44-71

22 Nilson T. A theoretical analysis of the frequency of gaps in plant stands. Agric Meteor, 1971, 8: 25-38

23 Kuusk A. The hot spot effect of a uniform vegetative cover. Sov J Remote Sens, 1985, 3: 645-658

24 间彬彦. 遥感反演叶面积指数的关键问题. 硕士学位论文. 北京: 北京大学, 2011.9-11

25 Fan W J, Xu X R, Liu X C, et al. Accurate LAI retrieval method based on PROBA/CHRIS data. Hydrol Earth Syst Sci, 2010, 14: 1499-1507

26 周秀䩀, 陶善昌, 姚克亚. 高等大气物理学(上下册). 北京: 气象出版社, 1991. 821-824

27 童庆禧, 张兵, 郑兰芬. 高光谱遥感——原理、技术与应用. 北京：高等教育出版社, 2006. 158-159

28 浦瑞良, 宫鹏. 高光谱遥感及其应用. 北京: 高等教育出版社, 2000. 52-53

29 Chen J M, Menges C H, Leblanc S G. Global mapping of foliage clumping index using multi-angular satellite data. Remote Sens Environ, 2005, 97: 447-457

30 武鹏飞, 宫辉力, 周德民. 近 30 年官厅水库流域景观结构的时空演变. 生态学杂志, 2011, 30: 2335-2342

31 杨胜天, 李茜, 刘昌明, 等. 应用“北京一号”遥感数据计算官厅水库库滨带植被覆盖度. 地理研究, 2006, 25: 570-577

32 刘晓臣. 基于多角度高光谱数据的植被 LAI 反演方法研究. 硕士学位论文. 北京: 北京大学, 2008 